\title{
Off-Line Parameter Identification of Permanent Magnet Synchronous Motor Using a Goertzel Algorithm
}

\author{
Jae-Seung Yoon*, Kyoung-Gu Lee**, June-Seok Lee* and Kyo-Beum Lee ${ }^{\dagger}$
}

\begin{abstract}
Generally, internal parameters of the motors and generators can be divided to the resistance and inductance components. They can become a cause of the changing internal parameters because they have sensitive characteristics due to external conditions. The changed parameters can generate the outputs which include error values from the speed and current controllers. Also, it can bring the temperature increase and mechanical damage to the system. Therefore, internal parameters of the motors and generators need to obtain their values according to the external conditions because it can prevent the mechanical damage caused by the changed parameters. In this paper, the off-line parameter identification method is verified using the Goertzel algorithm. The motor used in the simulation and experiments is an interior permanent magnet synchronous motor (IPMSM), and the proposed algorithm is verified by the simulation and experimental results.
\end{abstract}

Keywords: Off-line parameter identification, parameter estimation, PMSM, IPMSM, Goertzel algorithm

\section{Introduction}

Motors and generators are used in many areas such as robots, automobiles and ships which require reliability, efficiency, and precision. Motors and generators use controllers according to the purpose of the usage, and current and speed controllers are the most common controller among them. On the other hand, internal parameters of the motor and generator can be divided into the resistance and inductance components, and the current and speed controllers generate outputs according to each of the gain by internal parameters. However, these internal parameters have sensitive characteristics due to external conditions. Generally, internal temperature of motors and generators increases by the operation time, and it can change values of the internal parameters. Therefore, there is an error between the specification and actual parameters of the system, and it can bring the negative influence to the system. Also, temperature of the system can further be increased by error values of the controller, and it can generate the unbalanced power, mechanical vibration and a short-circuit between wires. Therefore, the motor control needs the information of changed internal parameters by the increased temperature for the high-efficiency control $[16,19]$.

The Goertzel algorithm is a method that performs the discrete Fourier transform (DFT) from the continuous

$\dagger \quad$ Corresponding Author: Dept. of Electrical and Computer Engineering, Ajou University, Suwon, Korea. (kyl@ajou.ac.kr)

* Dept. of Electrical and Computer Engineering, Ajou University, Suwon, Korea. (patorney@hanmail.net, junpb@ajou.ac.kr)

** Central R\&D Center, WOOJIN Industrial System Co., Ltd, 95, Korea. (kglee@wjis.co.kr)

Received: November 5, 2014; Accepted: May 27, 2015 signals. Generally, the fast Fourier transform (FFT) method has shorter calculation time than the DFT method. Hence, it is used often in the signal analysis method. However, the Goertzel algorithm has an advantage in the case of detecting numbers of smaller signals because it is faster than the FFT method. Also, it is possible to implement through the micro controller unit (MCU) of the low performance specification. Therefore, the Goertzel algorithm detects the high-frequency components of the voltage and current which can also be divided into real and imaginary components and can be expressed into the magnitude and phase of the high-frequency components $[1,3,5]$.

In this paper, parameters of the interior permanent magnet synchronous motor (IPMSM) are estimated using an off-line identification method. Also, the proposed algorithm is divided into the two modes. First, the stator resistance value is estimated. Generally, the stator resistance estimation mode is a method using the relationship expression of the voltage, current and resistance. The offline parameter identification method is the estimation method in standstill, and it is applied to the d-axis current of reactive component. Second, values of the d- and q-axes inductance are estimated. The inductance estimation mode used the Goertzel algorithm, and it is method that injects a high-frequency voltage. The high-frequency voltages are generated, and are injected into the d- and q-axes. Also, the injecting high-frequency voltages are analyzed to the Goertzel algorithm $[5,12]$.

Hence, the total impedance is calculated by magnitude and phase of d- and q-axis from the component of highfrequency and each of the d-and q-axis inductances is estimated by considering the iron-loss. The inductance estimation method is estimated in standstill. 
The off-line parameter identification method of an IPMSM using the Goertzel algorithm verifies feasibility through the simulation and experiment.

\section{Resistance Estimation}

\subsection{Modeling of IPMSM}

Fig. 1 shows the structure of an IPMSM. The IPMSM has different inductance of $\mathrm{d}$ - and q-axis depending on the length of the effective air-gap. The part having the pole in rotor is defined by d-axis, and it is flux generating direction by the field winding. The q-axis is defined to be in the direction of the electrical angle of $90^{\circ}$ from the d-axis.

Fig. 2 shows the equivalent circuit of the IPMSM structure, and the voltage equations for each d- and q-axes are shown in Eq. (1) and (2) [15-17].

$$
\begin{aligned}
& u_{d s}=R_{s} i_{d s}+\frac{d \lambda_{d s}}{d t}-\omega \lambda_{q s} \\
& u_{q s}=R_{s} i_{q s}+\frac{d \lambda_{q s}}{d t}+\omega \lambda_{d s},
\end{aligned}
$$

where, $u_{d s}$ and $u_{q s}$ are the injected d- and q-axis voltages, $i_{d s}$ and $i_{q s}$ are d- and q-axis currents, $R_{s}$ is the stator resistance, $\omega$ is the angler velocity and $\lambda_{\mathrm{ds}}$ and $\lambda_{\mathrm{qs}}$ are the $\mathrm{d}$ - and q-axis flux.

The back-EMF is defined in (3) because the proposed algorithm estimates the parameters in standstill.

$$
\begin{aligned}
& \omega \lambda_{d s}=0 \\
& \omega \lambda_{q s}=0 .
\end{aligned}
$$

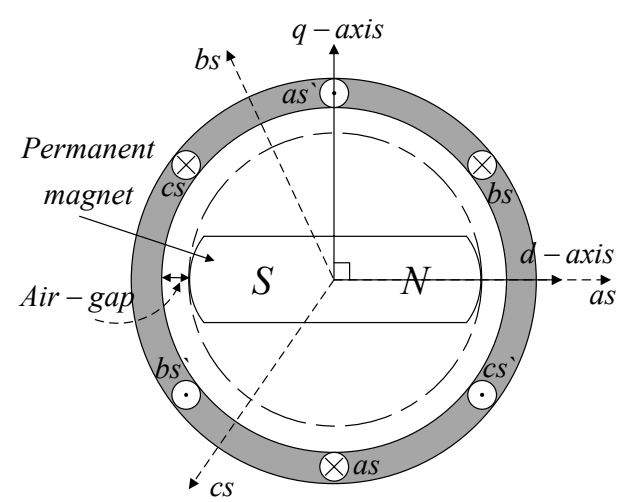

Fig. 1. Structure of the IPMSM.

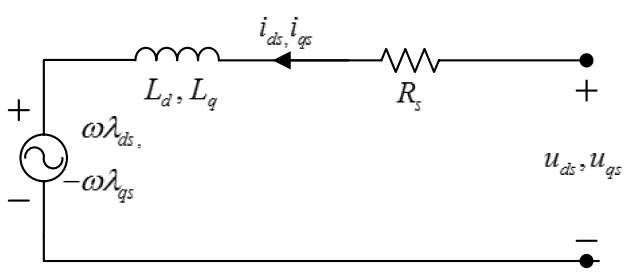

Fig. 2. Equivalent circuit of the IPMSM.

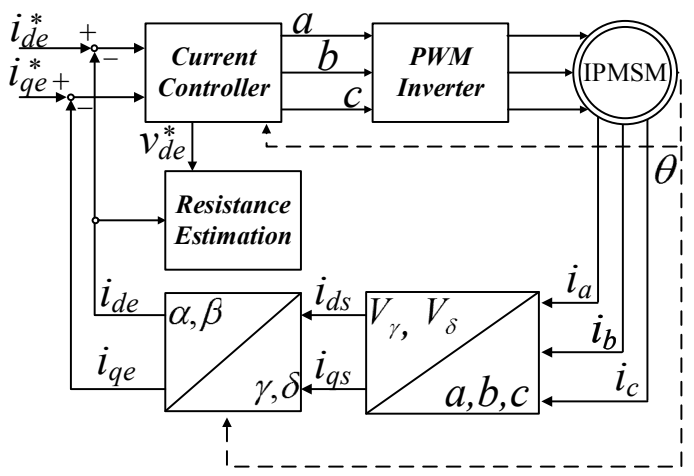

Fig. 3. Block diagram of the stator resistance estimation.

\subsection{Resistance estimation using d-axis current injection}

Fig. 3 shows the block diagram of the stator resistance estimation. The stator resistance estimation is composed of the coordinate transformation and current controller, and the current controller controls the current using a rotor position information obtained from an encoder.

Generally, the stator resistance can be estimated using a relationship between the voltage, current and resistance $[13,14]$. However, the estimated values include the error of the nonlinear voltage curve of insulated gate bipolar transistors (IGBTs). In the case of the stator resistance estimation, the input current which is close to the rated current has to be applied since the nonlinear voltage curve should be considered [8,9]. The stator resistance estimation is defined as

$$
\hat{R}_{s}=\frac{v_{d e}{ }^{*}}{i_{d e}},
$$

where the estimated $R_{s}$ is the estimated stator resistance, $v_{d e}{ }^{*}$ is the $\mathrm{d}$-axis synchronous reference frame voltage and $i_{d e}{ }^{*}$ is the $\mathrm{d}$-axis synchronous reference frame current.

In this paper, the proposed method is standstill of motor. Since the d-axis current is a reactive component which is in the flux generating direction, the d-axis current close to the rated value will not generate the speed $[3,4]$. Therefore, daxis reference current used the value close to rated current, and q-axis reference current used the value close to zero.

\section{Inductance Estimation}

\subsection{Relationship between flux and inductance}

The flux of the IPMSM is defined by the stator flux and flux by the permanent-magnet and which it is expressed as

$$
\lambda_{a b c s}=\lambda_{a b c s(s)}+\lambda_{a b c s(f)},
$$

where $\lambda_{a b c s(s)}$ is the linkage flux in the stator winding and 


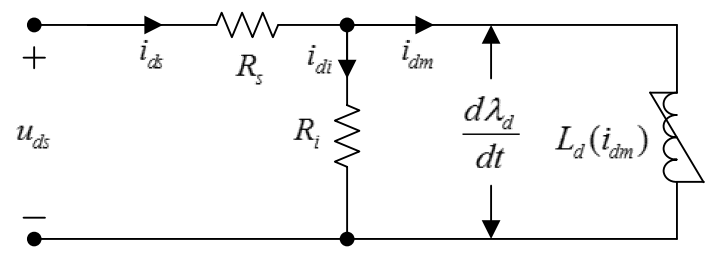

Fig. 4. d-axis equivalent circuit of the inductance.

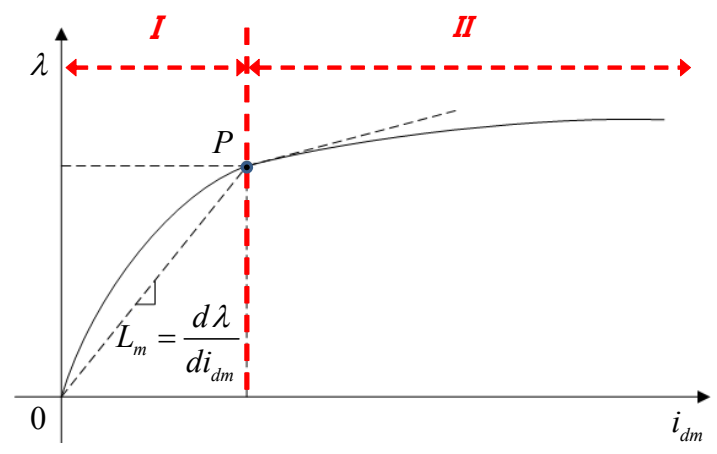

Fig. 5. Characteristic of the flux and current.

$\lambda_{a b c s(f)}$ is the linkage flux by the permanent-magnet.

Each flux of the 3-phase can be expressed to the d- and q-axes fluxes in (6).

$$
\begin{aligned}
& \lambda_{d s}=\left(L_{l s}+L_{m d}\right) i_{d s}+L_{s f} i_{f}=L_{d s} i_{d s}+\phi_{f} \\
& \lambda_{q s}=\left(L_{l s}+L_{m q}\right) i_{q s}=L_{q s} i_{q s}
\end{aligned},
$$

where $L_{l s}$ is leakage inductance, $L_{m}$ is magnetizing inductance of the d- and q-axis and $L_{s f}$ is inductance of permanent-magnet according to the position of rotor.

Fig. 4 is d-axis equivalent circuit of inductance. $i_{d i}$ is the iron-loss current, $R_{i}$ is the iron-loss resistance and $i_{d m}$ is the magnetizing current d-axis. Also, d-axis current is stationary coordinate and it is made up by the sum of the iron-losses current and magnetizing current.

Therefore, Fig. 5 can be separated by $I$ and $I I$ regions. $I$ region is the magnetizing part and its slope shows linear inductance. Also, $I I$ region is the leakage part and $P$ is the saturation point. The inductance in $I I$ region has a little value by saturation state.

Eq. (7) and (8) show the saturation and linear state of the flux by the magnetizing-current, respectively.

$$
\begin{gathered}
\lambda_{d}\left(i_{d m}(t)\right)=L_{m}\left(i_{d m}(t)\right) i_{d m}(t)+\phi_{f} \\
\frac{d \lambda_{d}\left(i_{d m}(t)\right)}{d t}=L_{d}\left(i_{d m}\right) \frac{d i_{d m}(t)}{d t} .
\end{gathered}
$$

Eq. (8) can be redefined to (9).

$$
L_{d}\left(i_{d m}\right)=\frac{d \lambda_{d}\left(i_{d m}(t)\right)}{d i_{d m}(t)}=\frac{d L_{m}\left(i_{d m}(t)\right)}{d i_{d m}(t)}
$$

Hence, the d-axis inductance by the magnetizing current is defined by (10).

$$
L_{d}\left(i_{d m}\right)=L_{m}\left(i_{d m}(t)\right)+i_{d m}(t) \frac{d L_{m}\left(i_{d m}\right)}{d i_{d m}} .
$$

However, the d-axis inductance of (10) does not consider iron-losses components. Therefore, iron-losses components need to be added in this equation to obtain the correct value of the d-axis inductance.

\subsection{Inductance estimation using goertzel algorithm}

FFT is popularly used in the signal analysis because it has a short calculation time. However, FFT requires the MCU of high-performance. On the other hand, the Goertzel algorithm is the DFT method. It has a long calculation time. However, the Goertzel algorithm has a short calculation time in the case of detecting the number of a small signal. Also, it is possible to implement through the MCU of low-performance. Table 1 shows the computation efforts of the Goertzel algorithm and FFT [19].

Table 1. Comparison of the computation effort $(\mathrm{N}$ is the number of sampling)

\begin{tabular}{c|c|c}
\hline Computation & Multiplies & Adds \\
\hline FFT & $\mathrm{N}^{2}$ & $\mathrm{~N}(\mathrm{~N}-1)$ \\
\hline Goertzel Algorithm & $\mathrm{N}+1$ & $2 \mathrm{~N}+1$ \\
\hline
\end{tabular}

The inputs of the Goertzel algorithm are the voltage and current of either the d-axis or q-axis. Fig. 6 shows the block diagram of the Goertzel algorithm [1-5].

In this paper, high-frequency is injected because the parameters are estimated in the standstill. Hence, the voltage and current include high-frequency. Eq. (11) defines high-frequency voltage $\left(V_{h f}\right)$.

$$
V_{h f}=V_{M a g, h f} \cos \left(\theta_{h f}\right) \text {, }
$$

where, $\theta_{h f}$ is angle of the high-frequency and $V_{M a g, h f}$ is the magnitude of high-frequency signal.

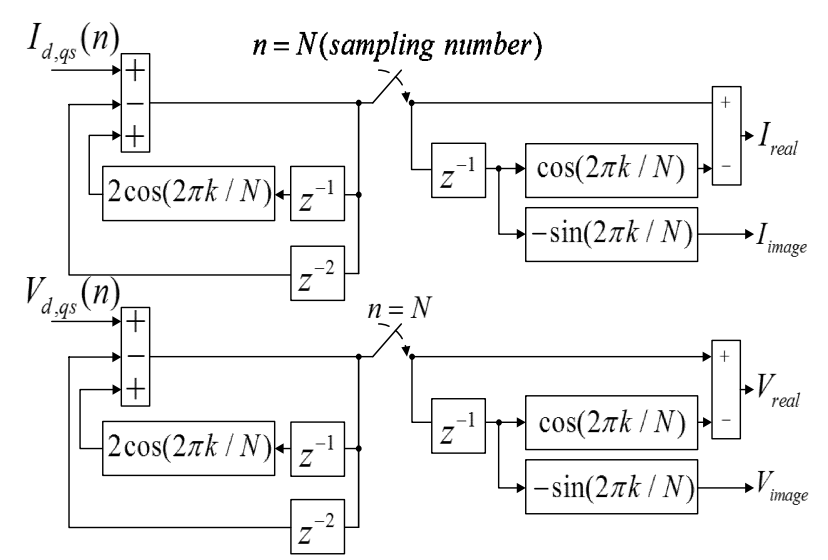

Fig. 6. Block diagram of the Goertzel algorithm. 
The inputs of the Goertzel algorithm are the voltage and current including the high-frequency. The Goertzel algorithm can obtain the real and imaginary components from the signal including high-frequency, and which analyzes the continuous signal during the sampling number $\mathrm{N}$. Hence, the outputs show the real and imaginary components. Also, the Goertzel algorithm can be indicate the magnitude and phase by the real and imaginary components, and the output is the same with an input highfrequency. However, the Goertzel algorithm includes errors in the magnitude and phase information because they include error values by $\mathrm{N}$ and the discrete time delay. Therefore, the Goertzel algorithm is required for selecting the appropriate number $\mathrm{N}$, and it needs to consider error values of the discrete time delay.

However, in this paper, the voltage and current of the proposed Goertzel algorithm have the same time delay. Also, the better resolution can be obtained with the higher $\mathrm{N}$, and $\mathrm{N}$ is selected from 50 to 100 considering the calculation time of a Texas Instruments TMS320F28335 digital signal controller (DSC) is used as the MCU.

To estimate the inductance, Inputs of the Goertzel algorithm are each of the current $\left(I_{d s}, I_{q s}\right)$ and voltage $\left(V_{d s}\right.$, $V_{q s}$ ) which contain high-frequency components. Inputs, in the case of the d-axis inductance estimation, are the d-axis stationary reference frame current and voltage, and are the q-axis stationary reference frame current and voltage in the case of the q-axis inductance estimation. The outputs of the Goertzel algorithm are the real and imaginary components as shown in Fig. 6. Fig. 7 shows the simulation results of the Goertzel algorithm and the extracted voltage and current consist of only the frequency component of 1000 $[\mathrm{Hz}]$.

By using the real and imaginary components $[4,5]$, the magnitude $\left(I_{M a g, h j}, V_{M a g, h f}\right)$ and phase $\left(\theta_{I, h f}, \theta_{V, h f}\right)$ are calculated as

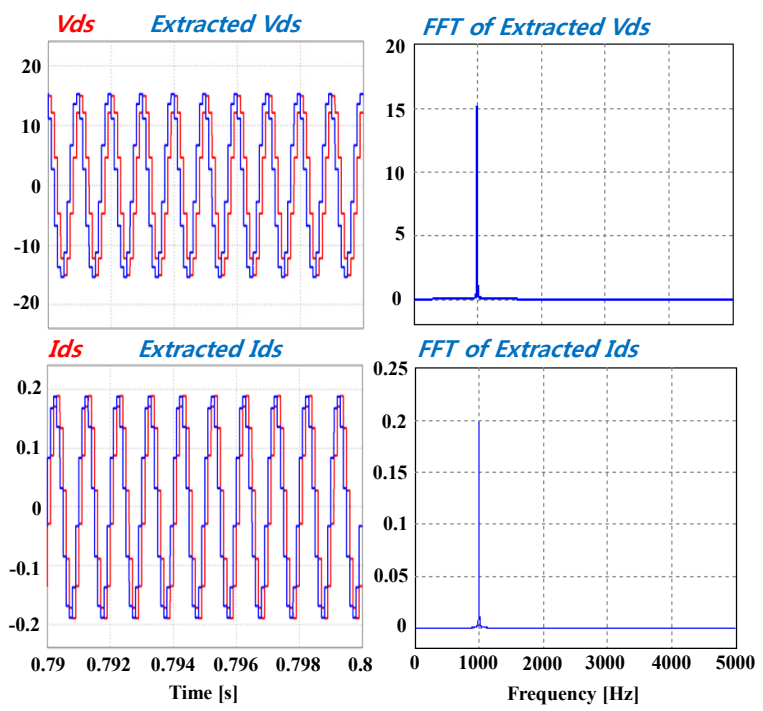

Fig. 7. Estimated results of high-frequency components from the Goertzel algorithm: $1000[\mathrm{~Hz}]$.

$$
\begin{gathered}
I, V_{\text {Mag }, h f}=\frac{\sqrt{\left(z^{-1}\right)^{2}+\left(z^{-2}\right)^{2}-\left(z^{-1} \times z^{-2} \times \cos 2\right)}}{(2 \times N)} \\
\theta_{I, h f}=\operatorname{atan} 2\left(I_{\text {image }}, I_{\text {real }}\right) \\
\theta_{V, h f}=\operatorname{atan} 2\left(V_{\text {image }}, V_{\text {real }}\right)
\end{gathered}
$$

where, $\cos =\cos \left(\theta_{h f} / d t\right), \cos 2=2 \times \cos$, and $\sin =\sin \left(\theta_{h f} / d t\right)$.

Also, the magnitude of (12) is without the dead time compensation. Hence, the valid voltage $\left(V_{\text {mag,hf,valid }}\right)$ is redefined as

$$
V_{\text {Mag, hf,valid }}=V_{\text {Mag, hf }}-V_{\text {Dead }} \text {. }
$$

$V_{\text {Dead }}$ is the compensation voltage of the dead time, and it is defined as

$$
V_{\text {Dead }}=\frac{V_{D C}}{2} \times \frac{T_{\text {Dead }}}{T_{s w}},
$$

where $V_{D C}$ is the DC link voltage, and $T_{s w}$ is the switching period. Also, $T_{\text {dead }}$ is the dead time and it was set $2[\mu \mathrm{sec}]$ [12].

The total impedance $(Z)$ can obtain from the magnitude and the phases of the voltage and current in the Goertzel algorithm. The total impedance $(Z)$ can be divided into the resistance $\left(Z_{R}\right)$ and inductance $\left(Z_{\omega L}\right)$ as shown in Fig. 8. Therefore, the magnitude $\left(Z_{M a g}\right)$ and phase $\left(\theta_{Z}\right)$ of the total impedance is defined as

$$
\begin{aligned}
& Z_{\text {Mag }}=\frac{V_{\text {Mag }, h f, v a l i d}}{I_{M a g, h f}} . \\
& \theta_{Z}=\theta_{I, h f}-\theta_{V, h f}
\end{aligned}
$$

The impedance of the real and imaginary components is defined as

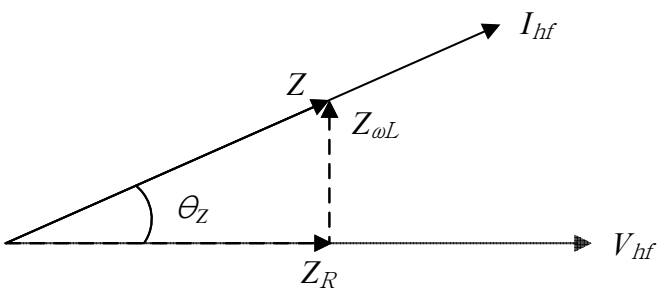

(a)

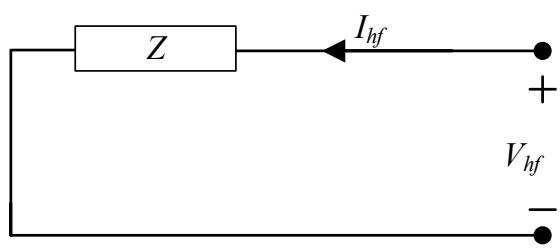

(b)

Fig. 8. Total impedance: (a) Vectors of impedance; (b) Equivalent circuit of impedance. 


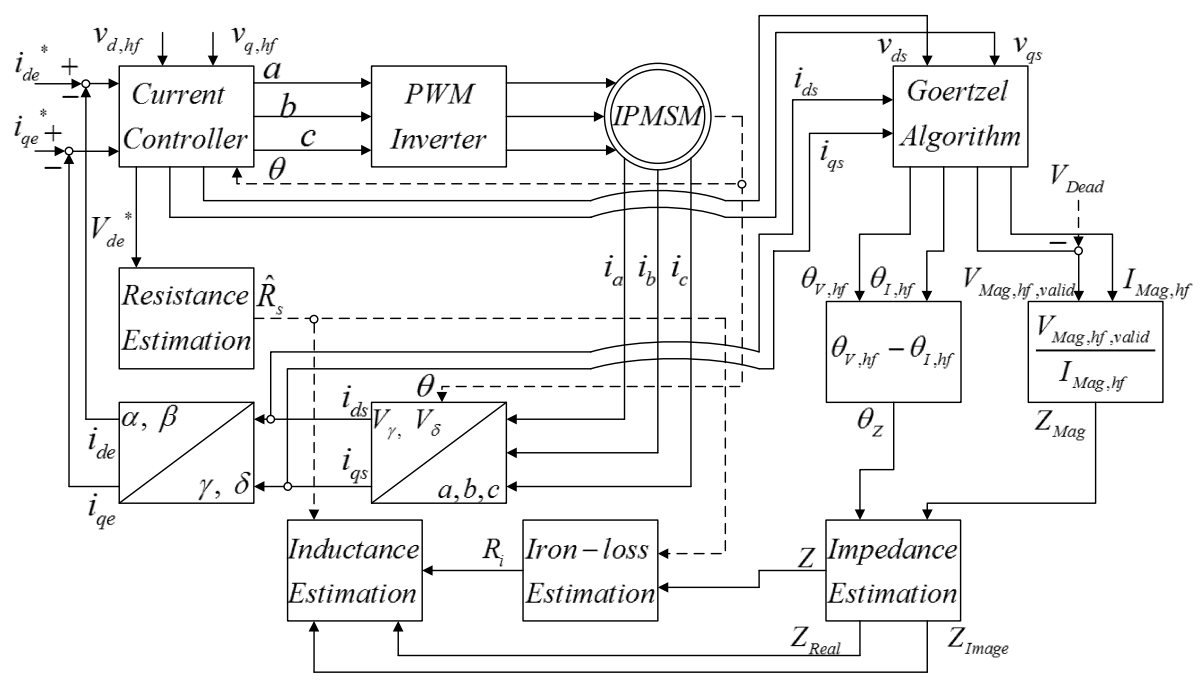

Fig. 9. The total block diagram of the proposed parameter estimation method.

$$
\begin{aligned}
& Z_{\text {real }}=Z_{\text {Mag }} \times \cos \left(\theta_{Z}\right) \\
& Z_{\text {image }}=Z_{\text {Mag }} \times \sin \left(\theta_{Z}\right)
\end{aligned} .
$$

The iron-loss is defined by (18) from the magnitude and phase of the impedance, and it is used to define the inductance by (19) [5-7].

$$
\begin{gathered}
R_{i}=\frac{\left(Z_{\text {real }}-\hat{R}_{s}\right)^{2}+Z_{\text {image }}{ }^{2}}{Z_{\text {real }}-\hat{R}_{s}} \\
\hat{L}_{d, q}=\frac{R_{i}\left(Z_{\text {real }}-\hat{R}_{s}\right)}{\omega_{h f} \times Z_{\text {image }}} .
\end{gathered}
$$

Fig. 9 shows the total block diagram of the proposed parameter estimation method.

\section{Simulation and Experimental Results}

\subsection{Simulation}

The simulation is performed using the PSIM tool. The rated power, speed and current (RMS) of the motor are 11 [kW], 1750 [rpm] and 19.9 [A] respectively. Table 2 shows parameters of the proposed parameter identification method, and Table 3 shows the motor specifications. The daxis reference currents are values close to the rated value in the case of the resistance estimation. However, d- and qaxes reference current are values close to zero in the case of the inductance estimation which applies the reference voltage of the high-frequency.

Also, the Goertzel algorithm can obtain better resolution by an increasing number of sampling, and outputs can bring better exact values. The simulation results of Fig. 10 select the frequency of $1000[\mathrm{~Hz}]$ and the number of the
Table 2. Parameters of the proposed algorithm

\begin{tabular}{c|c}
\hline Sampling Number $(\mathrm{N})$ of the Goertzel & 50 \\
\hline High-frequency $\left(\mathrm{f}_{\mathrm{hf}}\right)$ & $1000 \mathrm{~Hz}$ \\
\hline High-frequency voltage $\left(\mathrm{V}_{\mathrm{d}, \mathrm{qh}}\right)$ & $15 \mathrm{~V}$ \\
\hline Inductance estimation mode $\left(\mathrm{I}_{\mathrm{d}, \mathrm{q}}{ }^{*}\right)$ & $0 \mathrm{~A}$ \\
\hline Resistance estimation mode $\left(\mathrm{I}_{\mathrm{de}}{ }^{*}\right)$ & $24 \mathrm{~A}$ \\
\hline Control period & $100 \mu \mathrm{sec}$ \\
\hline
\end{tabular}

Table 3. Motor specifications

\begin{tabular}{c|c}
\hline Resistance $\left(\mathrm{R}_{\mathrm{s}}\right)$ & $0.349 \Omega$ \\
\hline $\mathrm{d}$-axis inductance $\left(\mathrm{L}_{\mathrm{d}}\right)$ & $13.17 \mathrm{mH}$ \\
\hline $\mathrm{q}$-axis inductance $\left(\mathrm{L}_{\mathrm{q}}\right)$ & $15.6 \mathrm{mH}$ \\
\hline Flux linkage & $0.554 \mathrm{wb}$ \\
\hline Number of poles & 6 \\
\hline Inertia & $0.02 \mathrm{~kg} \cdot \mathrm{m}^{2}$ \\
\hline Switching frequency $\left(\mathrm{f}_{\mathrm{sw}}\right)$ & $10 \mathrm{kHz}$ \\
\hline Rated power & $11 \mathrm{~kW}$ \\
\hline Rated current & $19.9 \mathrm{~A}$ \\
\hline
\end{tabular}

sampling is 50 .

In this paper, the proposed off-line parameter identification method was performed the simulation for the right calculation amount of MCU. Hence, the simulation was performed in the same condition as the experiment, and the validity of the proposed algorithm was verified by the simulation.

Fig. 10 shows the simulation result of the proposed parameter identification method. It shows estimated results of both resistance and inductance and these estimation modes can be checked through a flag sequence. The error range of parameter estimation is set to 5 [\%]. If the flag sequence is 1 , the proposed method of parameter identification estimates the value of stator resistance. Also, if the flag sequence is 2 , the proposed method of parameter identification estimates the values of $d-$ and qaxes inductance. The value of the estimated stator resistance can be retained during estimating mode of inductance. 


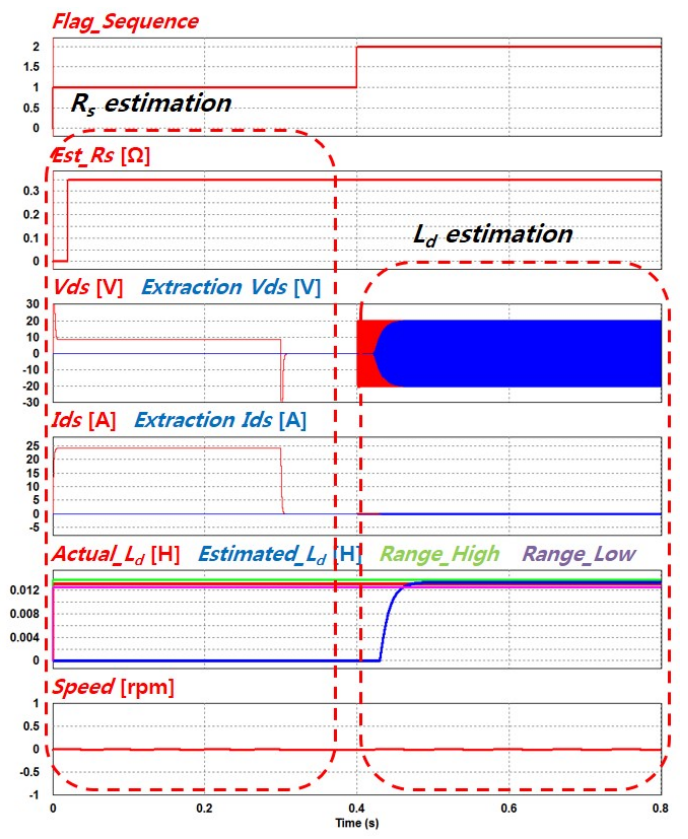

(a)

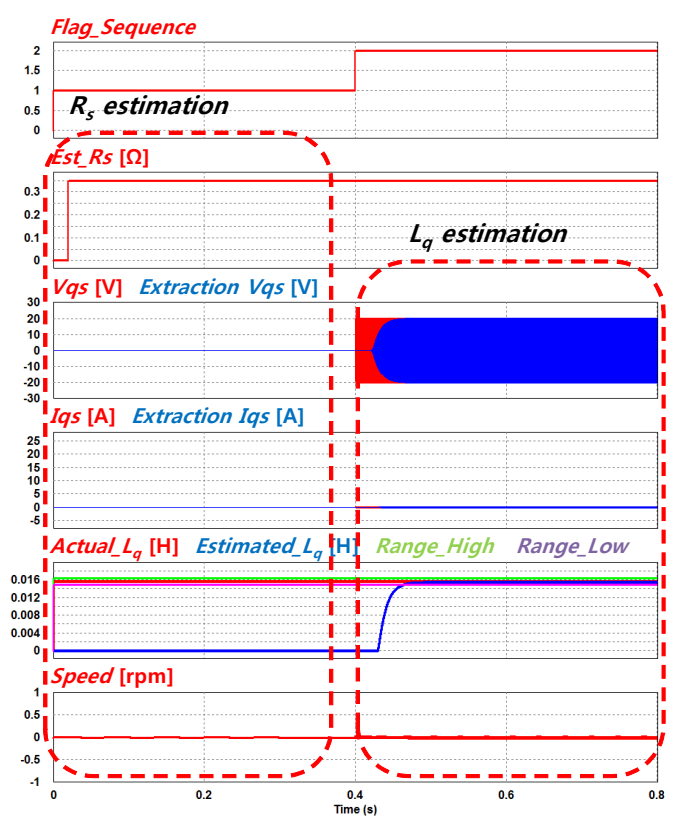

(b)

Fig. 10. The proposed parameter identification in standstill: (a) d-axis; (b) q-axis.

Further, estimating mode of inductance using an estimated value of stator resistance is conducted by using the highfrequency voltage and output of goertzel algorithm.

Fig. 11 shows the comparison result of the estimation performance according to the changing high-frequency. Fig. 11(a) is the case of d-axis, and (b) is the case of qaxis for the range of the changing frequency from 100 [Hz] to $1000[\mathrm{~Hz}]$. The d-axis inductance was the verified approximated value in the case of injected components of from 100 to $300[\mathrm{~Hz}]$, and the q-axis inductance was verified approximated value in the case of components

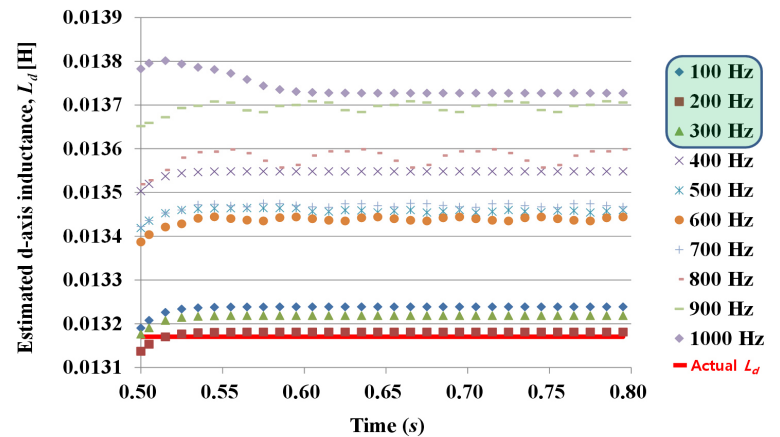

(a)

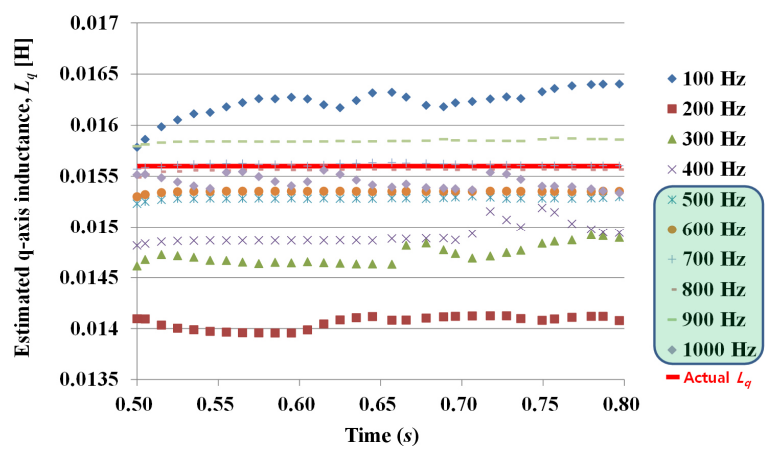

(b)

Fig. 11. Estimation performance according to the high frequency: (a) $L_{d}$; (b) $L_{q}$.

ranging from $500[\mathrm{~Hz}]$ to $1000[\mathrm{~Hz}]$. The performance of the Goertzel algorithm is determined by the high-frequency and the number of sampling. Therefore, the high-frequency is set such that it does not cause any influence to the fundamental frequency.

Also, the Goertzel algorithm can obtain better resolution by an increasing number of sampling, and outputs can bring better exact values.

In this paper, the proposed off-line parameter identification method was performed the simulation for the right calculation amount of MCU. Hence, the simulation was performed in the same condition as the experiment, and the validity of the proposed algorithm was verified by the simulation.

\subsection{Experimental results}

Fig. 12 shows the experimental set of a three-level NPC inverter, control board and M-G set.

Fig. 13 shows the resistance estimation. The estimated resistance values are used for the inductance estimation. Therefore, it should be an accurate estimation. Also, the MCU must consider the calculation time because it exceeds the calculation amount by the size of the array from the Goertzel algorithm.

Fig. 14 shows the high-frequency voltage analyzed from the Goertzel algorithm. The inputs of the Goertzel algorithm set by each of the $\mathrm{d}$ - or q-axis current and voltage. Figs. 14(a) and (b) each show the d- and q-axes 


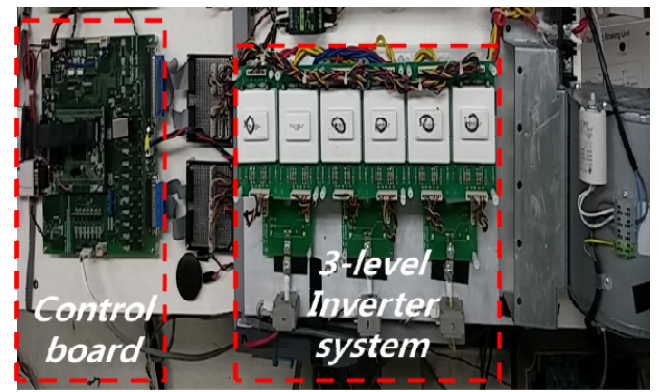

(a)

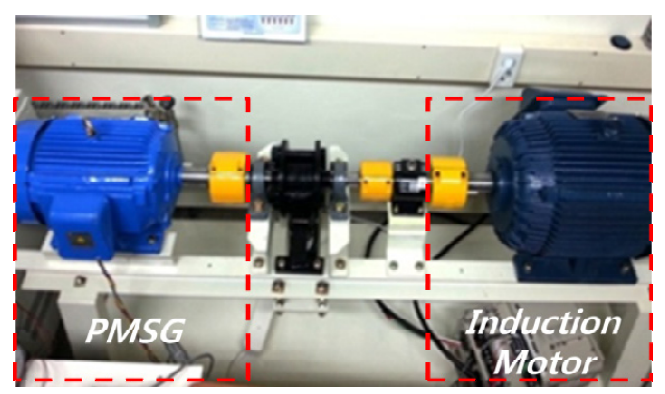

(b)

Fig. 12. The experiment equipment: (a) Three-level inverter and control board; (b) M-G set.

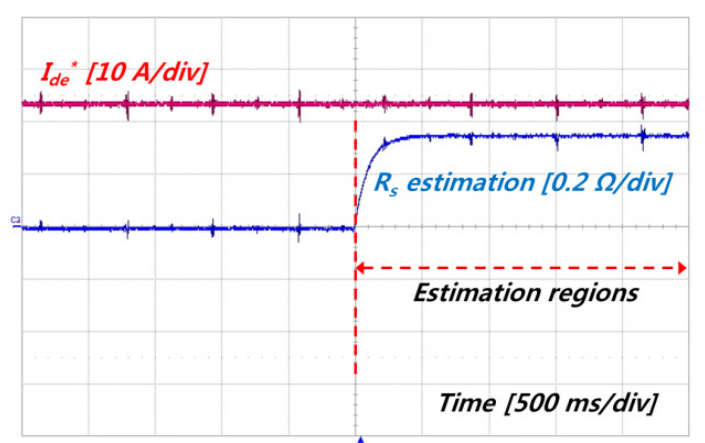

Fig. 13. Experimental result of the resistance estimation

high-frequency voltage, and also show the outputs of the analyzed result of the high-frequency voltages from the Goertzel algorithm.

Fig. 15 shows the estimated $\mathrm{d}$ - and q-axes inductance. Fig. 15(a) is the estimated d-axis inductance, and Fig. 15 (b) is the estimated q-axis inductance. The estimation method of inductance uses the estimated resistance value. The estimated resistance value is approximately $0.352[\Omega]$, and the estimated inductance includes some errors by the estimated resistance. Also, the estimated inductances are influenced by the high-frequency and resolution of the Goertzel algorithm. Therefore, the estimated value of $d-$ axis inductance is approximately $0.01321[\mathrm{H}]$ and the estimated value of $\mathrm{q}$-axis inductance is approximately $0.01554[\mathrm{H}]$.

Additionally, Fig. 16 shows the estimated d- and q-axes inductance in the switching frequency $1[\mathrm{kHz}]$. The control period was set to $500[\mu \mathrm{sec}]$. The initial values of $d-$ and

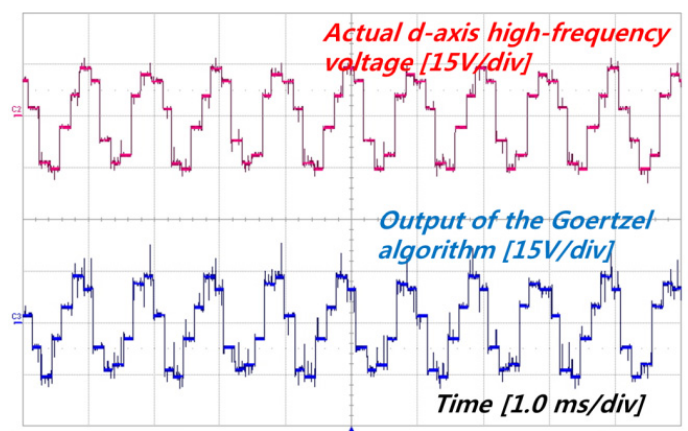

(a)

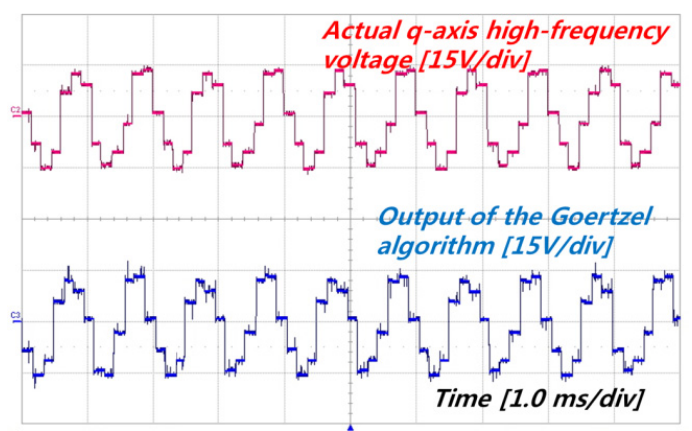

(b)

Fig. 14. High-frequency voltage analysis of the Goertzel algorithm: (a) d-axis; (b) q-axis.

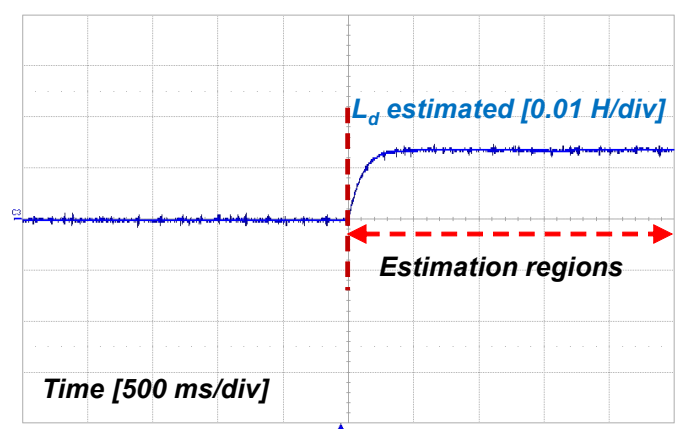

(a)

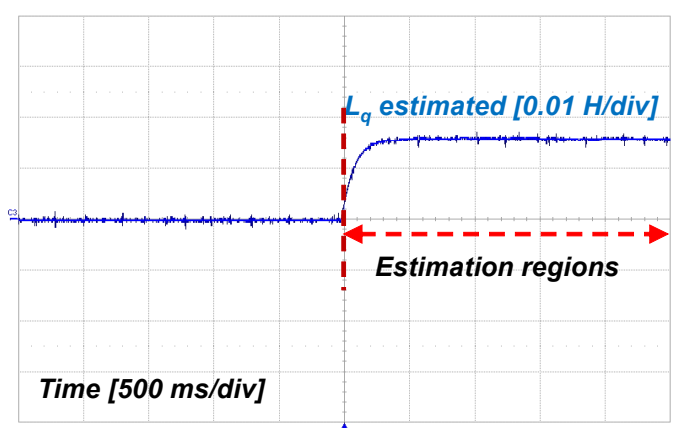

(b)

Fig. 15. Experiment result of the estimated inductance $\left(f_{s w}\right.$ : $10 \mathrm{kHz}$ ): (a) $L_{d}$; (b) $L_{q}$.

q-axes inductance are $50[\%]$ of the actual value. The experimental result of Fig. 16 shows performance of the 


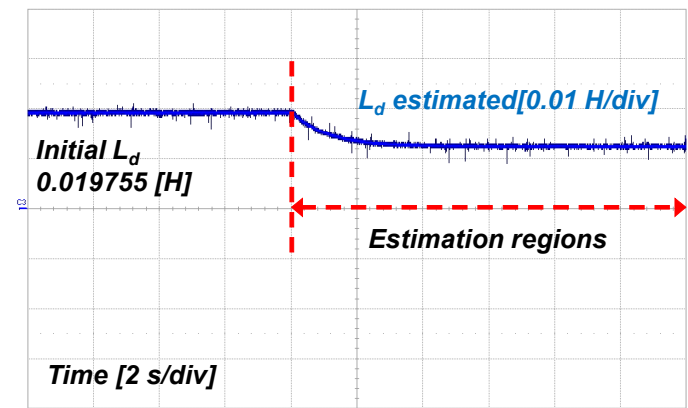

(a)

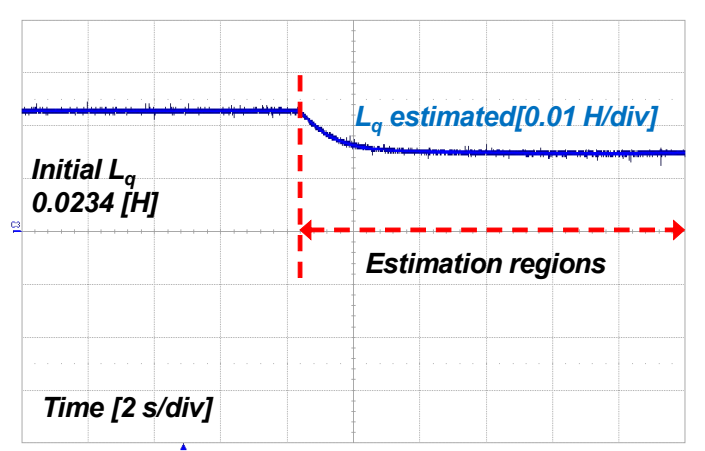

(b)

Fig. 16. Experiment result of the estimated inductance $\left(f_{s w}\right.$ : $1 \mathrm{kHz}$ ): (a) $L_{d}$; (b) $L_{q}$.

proposed parameter identification method by the initial values and switching frequency. The estimated result values of $\mathrm{d}$-axis inductance and q-axes inductance are approximately $0.01336[\mathrm{H}]$ and $0.01578[\mathrm{H}]$.

In this paper, the proposed parameter identification method in standstill was verified through the experimental results.

\section{Conclusion}

The proposed algorithm is divided into two modes in this paper. First mode estimates the stator resistance values and second mode estimates the inductance values. The stator resistance was estimated exactly in the first mode using a relationship between the voltage, current and resistance, and the first mode injects the d-axis current of the reactive component. Also, the q-axis current is the value close to zero. Hence, the d-axis reference current was set to the value close to the rated current, and the q-axis reference current was set to the zero value because the motor and generator are at standstill.

On the other hand, the second mode injected the highfrequency voltage, and analyzed the voltage and current of the $\mathrm{d}$ - and q-axes by the Goertzel algorithm. Hence, the second mode estimates the total impedance using a magnitude and phase of the high-frequency components. Also, this mode can obtain the iron-loss using an estimated total impedance, and it estimates inductance of the d- and q-axes using an outputs of the Goertzel algorithm.

In this paper, the performance of the proposed algorithm has been validated through the simulation and experimental results.

\section{Acknowledgment}

This work was supported by the Human Resources Development program (No.20134030200310) of the Korea Institute of Energy Technology Evaluation and Planning (KETEP) grant funded by the Korea government Ministry of Knowledge Economy.

This research was supported by Basic Science Research Program through the National Research Foundation of Korea (NRF) funded by the Ministry of Education (No. 2013R1A1A2A10006090).

\section{References}

[1] J. H. Kim, J. G. Kim, Y. H. Ji, Y. C. Jung, and C. Y. Won, "An Islanding Detection Method for a GridConnected System Based on the Goertzel Algorithm," IEEE Trans. Power Electron., vol. 26, no. 4, pp. 1049-1055, Apr. 2011.

[2] J. S. Lee, H. G. Jeong, and K. B. Lee, "Active Damping for Wind Power Systems with LCL filters using a DFT," Journal of Power Electron., vol. 12, no. 2, pp. 326-332, Mar. 2012.

[3] L. Peretti and M. Zigliotto, "Automatic Procedure for Induction Motor Parameter Estimation at Standstill," IET Electr. Power Appl., vol. 6, no. 4, pp. 214-224, Apr. 2012.

[4] R. Kerkman, J. Thunes, T. Rowan, and D. Schlegel, "A Frequency-Based Determination of Transient Inductance and Rotor Resistance for Field Commissioning Purposes," IEEE Trans. Ind. Appl., vol. 32, no. 3, pp. 577-584, May./Jun. 1996.

[5] N. Urasaki, T. Senjyu, and K. Uezato, "A Novel Calculation Method for Iron Loss Resistance Suitable in Modeling Permanent-Magnet Synchronous Motors," IEEE Trans. Energy Conv., vol. 18, no. 1, pp. 41-47, Mar. 2003.

[6] S. Morimoto, Y. Tong, Y. Takeda, and T. Hirasa, "Loss Minimization Control of Permanent Magnet Synchronous Motor Drives," IEEE Trans. Ind. Electron., vol. 41, no. 5, pp. 511-517, Oct. 1994.

[7] J. W. Choi and S. K. Sul, "Inverter Output Voltage Synthesis using Novel Dead Time Compensation," IEEE Trans. Power Electron., vol. 11, no. 2, pp. 221227, Mar. 1996.

[8] S. Bolognani, L. Peretti, and M. Zigliotto, "Repetitive-Control-Based Self-Commissioning Procedure for Inverter Nonidealities Compensation," IEEE Trans. Ind. Appl., vol. 44, no. 5, pp. 1587-1596, Sept./Oct. 
2008 .

[9] J. K. Seok, S. I. Moon, and S. K. Sul, "Induction Machine Parameter Identification using PWM Inverter at Standstill," IEEE Trans. Energy Conv., vol. 12, no. 2, pp. 127-132, Jun. 1997.

[10] A. Proca and A. Keyhani, "Identification of Variable Frequency Induction Motor Models From Operating Data," IEEE Trans. Energy Conv., vol. 17, no. 1, pp. 24-31, Mar. 2002.

[11] A. R. Munoz and T. A. Lipo, "On-line Dead-Time Compensation Technique for Open-Loop PWM-VSI Drives," IEEE Trans. Power Electron., vol. 14, no. 4, pp. 683-689, Jul. 1999.

[12] M. Hinkkanen, T. Tuovinen, L. Harnefors, and J. Luomi, "A Combined Position and Stator-Resistance Observer for Salient PMSM Drives: Design and Stability Analysis," IEEE Trans. Power Electron., vol. 27, no. 2, pp. 601-609, Feb. 2012.

[13] Y. S. Han, J. S. Choi, and Y. S. Kim, "Sensorless PMSM Drive with a Sliding Mode Control Based Adaptive Speed and Stator Resistance Estimator," IEEE Trans. Magn., vol. 36, no. 5, pp. 3588-3591, Sept. 2000.

[14] S. Po-ngam and S. Sangwongwanich, "Stability and Dynamic Performance Improvement of Adaptive Full-Order Observer for Sensorless PMSM Drive," IEEE Trans. Power Electron., vol. 27, no. 2, pp. 588600, Feb. 2012

[15] I. Jeong, B. J. Hyon, and K. Nam, "Dynamic Modeling and Control for SPMSMs with Internal Turn Short Fault," IEEE Trans. Power Electron., vol. 28, no. 7, pp. 3495-3508, Jul. 2013.

[16] H. W. Sim, J. S. Lee, and K. B. Lee, "On-line Parameter Estimation of Interior Permanent Magnet Synchronous Motor using an Extended Kalman Filter," Journal of Electrical Engineering \& Technology, vol. 9, no. 2, pp. 600-608, Mar. 2014.

[17] H. W. Sim, J. S. Lee, and K. B. Lee, "A Simple Strategy for Sensorless Speed Control for an IPMSM During Startup and Over Wide Speed Range," Journal of Electrical Engineering \& Technology, vol. 9, no. 5, pp. 1582-1591, Sept. 2014.

[18] A. Sarikhani and O. A. Mohammed, "Inter-Turn Fault Detection in PM Synchronous Machines by Physics-Based EMF Estimation," in Proc. ECCE, pp. 1254-1261, Sep. 2012.

[19] E. Jacobsen and R. Lyons, "The Sliding DFT," IEEE Signal Process. Mag., vol. 20, no. 2, pp. 74-80, Mar. 2003.

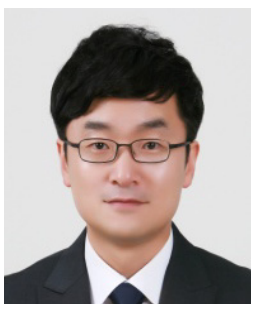

Jae-Seung Yoon received the B.S. and M.S. degrees in Electrical and Computer Engineering from the Ajou University, Korea, in 1996 and 2013, respectively. $\mathrm{He}$ is currently working toward the Ph.D. degree at Ajou University, Korea. His research interests include electric machine drives and switched reluctance motor drives.

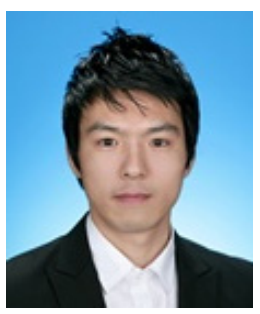

Kyoung-Gu Lee received his B.S. degree in Mechatronic Engineering from Korea Polytechnic University, Siheung, Korea, in 2012. He received his M.S. degree at Ajou University, Suwon, Korea, in 2015. He is assistant research engineer of the WOOJIN Industrial Systems Co., Ltd. Korea. His research interests include electric machine drives, switched reluctance motor drives and energy storage system.

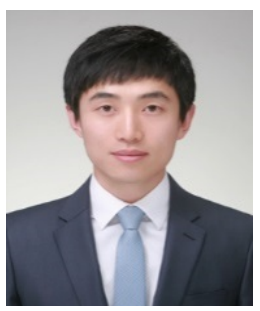

June-Seok Lee received the B.S. and M.S. degrees in Electrical and Computer Engineering from the Ajou University, Korea, in 2011 and 2013, respectively. He is currently working toward the Ph.D. degree at Ajou University, Korea. His research interests include grid-connected systems, multilevel inverter and reliability.

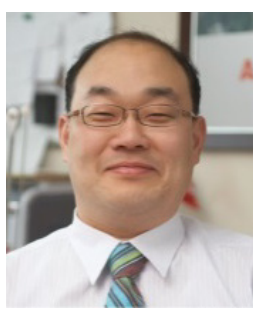

Kyo-Beum Lee received the B.S. and M.S. degrees in electrical and electronic engineering from the Ajou University, Korea, in 1997 and 1999, respectively. He received the $\mathrm{Ph} . \mathrm{D}$. degree in electrical engineering from the Korea University, Korea in 2003. From 2003 to 2006, he was with the Institute of Energy Technology, Aalborg University, Aalborg, Denmark. From 2006 to 2007, he was with the Division of Electronics and Information Engineering, Chonbuk National University, Jeonju, Korea. In 2007 he joined the School of Electrical and Computer Engineering, Ajou University, Suwon, Korea. He is an associated editor of the IEEE Transactions on Power Electronics, the Journal of Power Electronics, and the Journal of Electrical Engineering \& Technology. His research interests include electric machine drives, renewable power generations, and electric vehicle applications. 